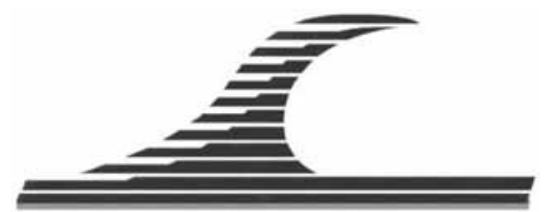

\title{
Apport de la sismique marine très haute résolution à l'étude de la rade abri de Brest (France)
}

\author{
Agnès BALTZER ${ }^{1}$, Roland BOUTIN ${ }^{2}$, Alexis STEPANIAN ${ }^{3}$
}

1. Université de Caen, Faculté des Sciences, UMR 6143 CNRS - M2C, Esplanade de la Paix, 14032 Caen, France.

agnes.baltzer@unicaen.fr

2. Direction des travaux maritimes de la région atlantique. Le Château, BP 16, 29240 Brest Armées, France. roland.boutin@wanadoo.fr

3. BRGM, Service Géologique Régional Provence Alpes Côte d'Azur (SGR/PAC), 117 Avenue de Luminy, B.P. 168, 13276 Marseille cedex 09, France.

a.stepanian@brgm.fr

\section{Résumé :}

Dans le cadre de la modernisation du port militaire de Brest, deux reconnaissances géophysiques réalisées avec le boomer Seistec (en 2004 et en 2006) ont permis de reconnaître la couverture sédimentaire et la profondeur du socle. De précédentes investigations avaient établi l'existence de décalages verticaux des couches géologiques grâce à des sondages. La vision "en continu" fournie par les profils sismiques THR permet d'appréhender de façon différente ces décalages, et de les associer à des structures ondulées remarquables, relatives à une phase de remplissage de la Baie. Cette vision globale des fonds permet de comprendre l'évolution géomorphologique de la rade dans son ensemble et de dépasser la vision "ponctuelle". Ainsi, l'influence de l’Aulne, et non de la Penfeld comme le laissait supposer la disposition actuelle, s'avère prépondérante.

Soumis le 23 février 2009, accepté le 19 octobre 2009, en ligne le 25 janvier 2010.

La seule version examinée est celle écrite en français. La ou les autres versions n'étant pas examinées par le comité de rédaction de la revue, sont donc publiées sous l'entière responsabilité du ou des auteurs. 


\section{Introduction}

Les reconnaissances géophysiques réalisées à l'aide du boomer Seistec avaient pour but d'acquérir le maximum d'information sur l'état des fonds marins dans la "rade abri" du port militaire de Brest (Bretagne, France), dans l'optique de travaux de réaménagements. Il s’agissait ainsi de compléter des études menées à partir de forages par la Société SIF BACHY (1966) et de sondages et d'essais in situ au pénétromètre dynamique, réalisés par l'entreprise Ménard en 1967.

Il s'avérait particulièrement important de répondre à deux questions : quelle était la profondeur du socle rocheux dans cette rade abri, et que représentaient les décalages verticaux décrits (MENARD, 1967) à partir de forages - étaient-ce des failles ou d'autres types de structures morphologiques ? En effet, cette zone est proche de la grande zone de faille du système de l'Elorn et l'on observe des anomalies topographiques à l'entrée de la Penfeld (HALLEGOUET, 1989).

L'acquisition de profils sismiques a permis d'aboutir à une vision dans le plan vertical, caractérisée par une très haute résolution. Cette approche facilite la compréhension des processus de mise en place des dépôts sédimentaires et donc de l'évolution de la morphologie des fonds marins.

\section{La sismique Très Haute Résolution}

Le boomer IKB-Seistec (www.seistec.ca/boomer.html) a été acquis par le laboratoire de Morphologie Continentale et Côtière de l'Université de Caen, dans le cadre de la mise en place du Pôle Géophysique Très Haute Résolution de Normandie (BALTZER et al., 2003).

Le principe de la prospection sismique des domaines sous-marins repose sur l'étude des échos d'une onde acoustique émise artificiellement et réfléchis par des interfaces : le fond de la mer et les limites entre les différentes couches sous-jacentes. Un réflecteur acoustique matérialise ainsi une interface (une différence d’impédance acoustique) lorsque l'onde émise traversant un milieu caractérisé par une impédance acoustique Z1 (Z1 = V1 * D1), aborde un nouveau milieu caractérisé par une nouvelle impédance acoustique Z2 (Z2 = V2 * D2). V1 et V2 sont les vitesses du son dans le milieu traversé, et D1 et D2 la densité respective de chacun de ces milieux. Lorsque les ondes émises sont de faible puissance et de très haute fréquence (quelques 10 à quelques $100 \mathrm{kHz}$ ), elles ne peuvent pas pénétrer dans le sédiment et sont totalement réfléchies par le fond de la mer seulement.

Plus les ondes émises sont de forte puissance et de basse fréquence (quelques $10 \mathrm{~Hz}$ à quelques $\mathrm{kHz}$ ), plus elles pénètrent profondément dans les sédiments et restituent ainsi la géométrie interne des différentes couches qui les composent. C'est le principe de la sismique. Au contraire de la sismique dite "conventionnelle" (recherche pétrolière) caractérisée par une forte pénétration (quelques $\mathrm{km}$ ) mais une basse résolution (quelques dizaines de mètres), la sismique utilisée pour étudier les domaines littoraux, 
où l'épaisseur des sédiments reste inférieure en général à la centaine de mètres, est dite "Très Haute Résolution" ou sismique THR. Elle répond à une double nécessité : l'étude à grande échelle des sédiments (résolution de l'ordre de $25 \mathrm{~cm}$ ) ainsi qu'une mise en oeuvre de l'outil dans des environnements peu profonds c'est-à-dire à partir de petites embarcations.

Le Seistec-IKB, fabriqué au Canada (SIMPKIN \& DAVIS, 1993 ; MOSHER \& SIMPKIN, 1999 ; SIMPKIN, 2005) est caractérisé par une source d'émission électromécanique : une plaque (un boomer) se déforme sous l'effet d'une décharge électrique de haute tension de 4000 Volts. Les échos de l'onde émise sont réceptionnés par des hydrophones placés prés de la plaque. Sa fréquence d'émission recouvre une bande de 1 à $10 \mathrm{kHz}$, pour une durée d'enregistrement du signal de retour variant de 75 à $250 \mathrm{~ms}$, en fonction de la profondeur d'eau et du pas de tir $(75 \mathrm{~ms}$ pour un pas de 4 ou 6 tirs par secondes et $250 \mathrm{~ms}$ pour un pas de 3 tirs par seconde). Nous l'avons utilisé avec une "puissance" de $150 \mathrm{~J}$ permettant une profondeur de pénétration d'une vingtaine de mètres dans la zone prospectée. Nous avons ainsi réalisé 48 profils sismiques dans la rade abri du port militaire, ils permettent d'obtenir une vision générale du socle et de sa couverture sédimentaire.

\section{Interprétation}

\subsection{Etat des connaissances}

Nous avions à notre disposition deux rapports basés sur des forages réalisés entre 1966 et 1967 afin d'étudier les caractéristiques géotechniques du sol de la rade : les deux premiers par la société SIF BACHY (1966) et le dernier par MENARD (1967).

Le premier rapport décrit 10 forages réalisés dans le sud-ouest de la rade, contre le digue sud (figure 1). Ces forages présentent tous une succession relativement similaire de deux couches de sable fin, gris plus ou moins coquiller, sur 3 à $5 \mathrm{~m}$ d'épaisseur reposant sur le substratum de schistes situé entre 4 et 7,5 $\mathrm{m}$ de profondeur. Une couche de vase noirâtre, de $30 \mathrm{~cm}$ à $2 \mathrm{~m}$ recouvre toute cette zone. Le second rapport décrit 3 forages réalisés dans le nord de la rade au niveau de l'épi de la grande rivière (appelé aussi épi de Laninon - figure 1). Le toit du schiste passe de 4,25 m à $6,50 \mathrm{~m}$ puis $8 \mathrm{~m}$ d'est en ouest. Une couche épaisse de sable et galets d'une épaisseur variant de 4 à $6 \mathrm{~m}$ recouvre ce substratum; elle est elle-même nappée par du sable vaseux en surface.

Le rapport de MENARD (1967) montre 7 coupes géologiques réalisées à partir de 58 forages, tout autour de la pointe (figure 1). On retrouve dans chaque cas le toit du schiste sur lequel repose une couche d'alluvions anciennes, variant de 1 à $5 \mathrm{~m}$. Ces alluvions anciennes sont composées de blocs de schistes, rognons de quartz et granite englobés dans une gangue de sable argileux. Une couche d'alluvions modernes, sable vasard avec passées de graviers, recouvre la série, sur une épaisseur de 4 à $5 \mathrm{~m}$ 
généralement, atteignant exceptionnellement $10 \mathrm{~m}$ et plus, sur les coupes 4, 5 et 7 .

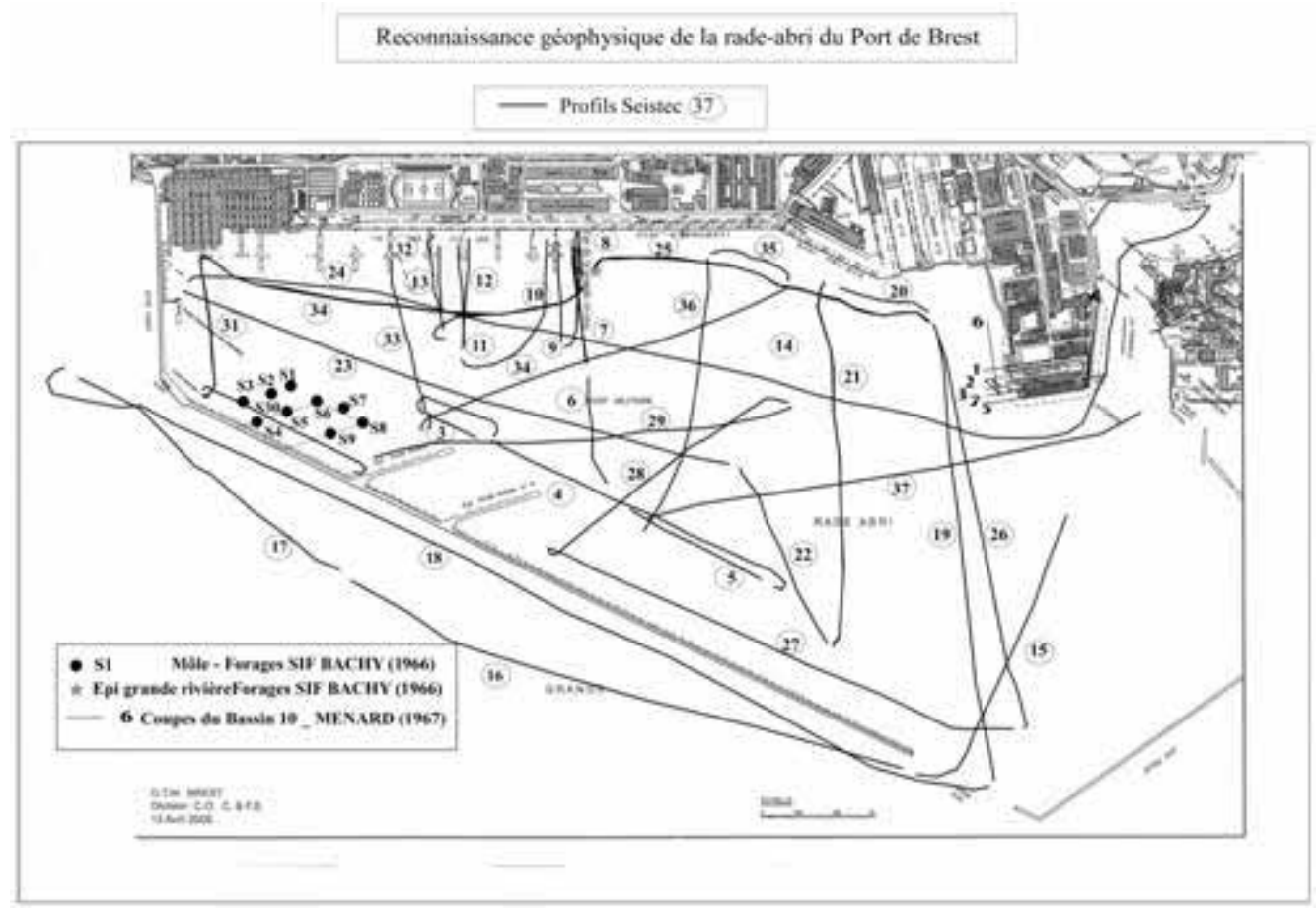

Figure 1. Localisation des sondages et des profils sismiques.

\subsection{Données sismiques et interprétation}

La figure 1 montre la répartition des 37 profils sismiques (du profil S1 à S37) réalisés dans la rade abri du port militaire de Brest. Ces profils recoupent la rade suivant plusieurs directions, permettant d'établir le contexte morphologique général.

En sismique réflexion les temps de parcours sont exprimés en millisecondes temps double (ms TD), c'est-à-dire le temps que met l'onde acoustique pour faire le trajet aller/retour entre l'hydrophone et l'interface sédimentaire. Pour en déduire une profondeur en mètres, il suffit de diviser par 2 cette échelle (temps d'un seul trajet) puis de multiplier par la vitesse du milieu traversé : $1500 \mathrm{~m} / \mathrm{s}$ dans l'eau et jusqu'à $2200 \mathrm{~m} / \mathrm{s}$ pour les sédiments marins superficiels en fonction de la nature des sédiments (voir tableau 1).

Dans la rade abri, la plupart des profils sont caractérisés par une pénétration maximum de $8 \mathrm{~ms}$ temps double, soit une pénétration maximum de $6,5 \mathrm{~m}$. Nous attirons ici l'attention sur le fait que si l'on considère une vitesse de $1800 \mathrm{~m} / \mathrm{s}$ ou $2200 \mathrm{~m} / \mathrm{s}$ nous atteignons des profondeurs de pénétration de l’ordre de 7,20 m ou de 8,8 m. 
Apport de la sismique marine très haute résolution à l'étude de la rade abri de Brest (France) : 1.5

Tableau 1. Corrélation vitesses sismiques et formations sédimentaires d'après le rapport FUGRO (1999) à partir de données de sismique réfraction et de carottages.

\begin{tabular}{ll}
\hline Vitesse sismique (m/s) & Formation géologique \\
\hline $1480-1600 \mathrm{~m} / \mathrm{s}$ & $\begin{array}{l}\text { Sables silteux ou argileux 'vasards”, sables pouvant être graveleux - } \\
\text { état lâche }\end{array}$ \\
$\begin{array}{l}\text { Sables moyennement denses à assez denses, pouvant contenir graviers } \\
\text { et galets, graviers avec galets possibles à l'état plus ou moins lâche. }\end{array}$ \\
$\begin{array}{l}\text { Sables et sables graveleux denses, graviers avec éventuellement galets } \\
1800-2000 \mathrm{~m} / \mathrm{s}\end{array}$ \\
\begin{tabular}{l} 
Graviers avec galets plus denses \\
\hline
\end{tabular}
\end{tabular}

Les profils montrent une succession de 5 faciès acoustiques (figure 2) que nous décrirons en commençant par le faciès le plus profond (donc le plus ancien), pour remonter vers la surface (le plus jeune). Ces faciès sont différenciés selon les règles de la stratigraphie sismique (MITCHUM et al., 1977) et constituent 5 unités sismiques surmontant le substratum rocheux qui sont décrites dans différents articles scientifiques sur les vallées incisées en Bretagne sud (PROUST et al., 2001 ; JOUET et al., 2003 ; MENIER et al., 2006).

Le substratum rocheux (vert foncé) : ce premier faciès acoustique "sourd", c'est-à-dire sans réflecteur visible, montrant des réflexions chaotiques et discontinues, correspond au toit du substratum rocheux des schistes à pendage vertical. Il constitue la base de tous les profils sismiques, décrit comme plus ou moins altéré dans sa partie supérieure, il laisse place aux schistes sains dans la partie sud (digue sud). Ce faciès correspond vraisemblablement aux schistes briovériens reconnus en Baie de Douarnenez (JOUET et al., 2003).

L'Unité $U^{r}$ : elle correspond à un faciès plus ou moins chaotique et transparent, d'une épaisseur variant de quelques mètres à 5 mètres maximum. Localisée uniquement dans la paléo-vallée de l'Aulne, cette unité est probablement constituée de sédiments grossiers et hétérogènes qui expliqueraient le caractère chaotique du faciès acoustique, et constituerait la première phase de remplissage de cette vallée incisée, semblable au remplissage des vallées incisées reconnu par JOUET et al. (2003) dans la Baie de Douarnenez.

L'Unité U1B (beige moiré) : cette unité d'une épaisseur de 3 à $5 \mathrm{~m}$ est constituée par un ensemble de réflecteurs concordants, continus, horizontaux déposés sur le substratum schisteux. Elle se retrouve dans toute la rade, sauf au sud, près de la digue sud où repose le banc de sable de St Pierre. Ce dépôt est ainsi $a$ priori synchrone ou juste postérieur à la mise en place de ce banc de sable. Cette unité correspond aux alluvions anciennes décrites par MENARD (1967): blocs de schistes et rognons de quartz (érosion 
continentale) entourés d'une gangue de sable argileux. Il est également possible de voir des "pockmarks" ou des remontées de fluides sur le profil S19, en bordure de la paléovallée de l'Aulne.

L'Unité U1A (jaune). Cette unité révèle un faciès acoustique transparent avec quelques réflexions chaotiques que l'on ne retrouve que dans le sud de la rade. Par endroit, il est possible de discerner des réflecteurs épais subverticaux (foresets) qui marquent la progradation de ce corps sédimentaire. Cette signature acoustique presque transparente (profils S4 et S27) correspond à l'existence de sable, en accord avec les dépôts de "sables gris fins et parfois vaseux et coquilliers" décrits dans les forages de SIF BACHY (1966). Cette unité U1A constitue le haut fond associé au banc de St Pierre, banc sableux sur lequel repose la digue sud.

L'Unité U2 (rouge) : c'est un faciès lité montrant plusieurs réflecteurs continus sur une épaisseur d'environ 5 à $6 \mathrm{~m}$. Ce faciès lité nappe les incisions faites dans le dépôt précédent d'alluvions anciennes. Le toit de ce faciès est à son tour érodé et incisé. Ces sédiments correspondent à une alternance de sables vasards et de graviers décrits par MENARD (1967) qui nappent la surface de discontinuité et présentent des ondulations sur les profils S37 et S19 (figure 3). L'unité de remplissage U2 est uniquement préservée dans cette cuvette, à l'ouest de l'éperon rocheux, à la sortie de la Penfeld (figure 5).

L’Unité U3 (orange) : ce faciès acoustique présente des réflexions chaotiques et recouvre les dépôts de U3 et U2. Il correspond aux alluvions modernes (sables vasards et passées graveleuses) provenant de l’Aulne, décrites par MENARD (1967). Il a donc été déposé après une période d'arrêt de la sédimentation qui se marque sur les profils par une surface d'érosion particulièrement visible en sommet de l'unité U4. Cette nouvelle phase de remplissage correspond à l'établissement des conditions actuelles de la sédimentation sous influence tidale marine.

L'Unité U4 (bleu) : c'est un faciès plus ou moins lité recouvrant l'Unité U4 correspondant à la vase noirâtre décrite dans la partie sud de la rade (proche de la digue). Nous observons un passage latéral de faciès de vase à un faciès plus sablovaseux qui correspond à la couverture superficielle que l'on rencontre dans la moitié nord de la rade. Cette différence de sédiments superficiels peut s'expliquer probablement par l'hydrodynamisme de la rade. La partie sud, plus confinée et plus fréquentée, permet le dépôt de vase, tandis que dans la moitié nord, les fines sont plus facilement remobilisées par les courants et le sable est ainsi "lavé" de ses fines.

Enfin, il faut noter la présence du paléo-lit de l'Aulne, parfaitement visible sur la figure 2, qui longeait la falaise dans les temps géologiques. Cette paléo-vallée divise la rade en deux, avec le banc St Pierre dans sa partie sud et les banquettes d'alluvions dans la moitié nord. 


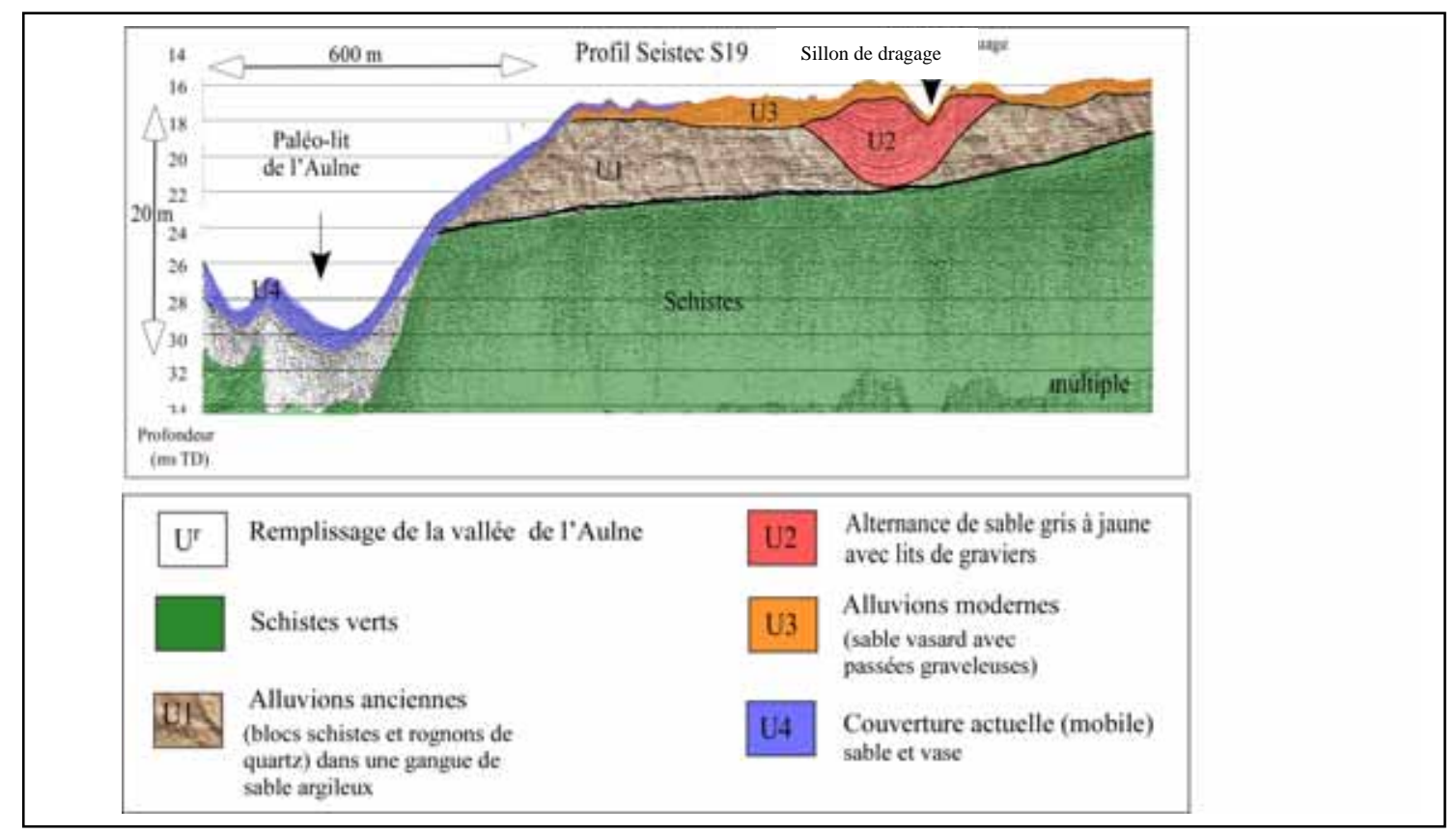

Figure 2. Profils sismiques Seistec révélant les différents faciès acoustiques.

\section{Discussion}

Grâce à la vision générale donnée par cette reconnaissance géophysique, il est possible de mieux suivre l'évolution morphodynamique des fonds marins de la rade abri. Elle est divisée en deux dans le sens de la longueur, par le passage d'une paléo vallée, qui n’est pas celle de la Penfeld comme on aurait pu le supposer, mais celle de l'Aulne, venant du fond de la grande rade de Brest (figure 6). La paléo vallée de l'Aulne rentre par la passe sud dans la rade, se prolonge jusque dans le fond de la rade abri et ressort au niveau de la digue ouest (zone de l'ancienne passe ouest). Elle présente les mêmes caractéristiques morphologiques, $600 \mathrm{~m}$ de large sur $20 \mathrm{~m}$ de profondeur, que les vallées incisées du système armoricain reconnu en sud bretagne (JOUET et al., 2003). Au sud de cette paléo vallée, le banc de St Pierre (support de la digue Sud) est visible sur le profil sismique S37 (figure 5). Au nord les dépôts sont constitués par des unités de remplissage sédimentaire.

Il est possible d'interpréter ces profils suivant 2 scénarios. La seule possibilité de les départager serait un carottage calibrant les différents faciès avec des datations. Nous exposerons donc ici les 2 scénarios possibles :

\section{Scénario 1 :}

Le comblement de la baie se déroule en 4 phases :

Phase 1 : une première phase de remplissage $\left(\mathrm{U}^{\mathrm{r}}\right)$ de la paléo vallée de la Penfeld qui correspond au remplissage des grandes vallées incisées du système armoricain (JOUET et al., 2003) lors d'une première remontée du niveau marin. Le niveau marin reste bas. 
Phase 2 : une seconde phase de remplissage s'établit dans le domaine estuarien de la Baie de Brest qui permet le dépôt de l'unité U1 (alluvions anciennes) sur les bords de la vallée de l'Aulne (MARTY, 1994). Ce remplissage atteste d'une nette élévation du niveau marin, ainsi que de la mise en place d'une dynamique tidale (établissement d'une barre sableuse) en domaine estuarien. Deux explications peuvent rendre compte de l'absence de U1 au dessus de $\mathrm{U}^{\mathrm{r}}$ sur le profil 19, soit un non dépôt de cette unité dû à un fort courant (hydrodynamisme exacerbé en raison des nouvelles conditions) soit à l'érosion du dépôt de U1 corrélée à une phase d’érosion générale observée dans la Baie. En effet, la phase de remplissage U1 est interrompue par un événement ou une phase d'érosion qui va entailler et creuser une dépression jusqu'au toit du schiste (figures 3, 4 et 5). Cette dépression ou cuvette sera ensuite comblée par un dépôt sédimentaire sableux (U2).

De nouveaux deux hypothèses peuvent expliquer cette phase d'érosion :

a) une érosion liée à un événement de type catastrophique et court dans le temps : une crise climatique caractérisée par un régime de tempêtes qui pourraient avoir creusé de profondes cicatrices et cuvettes dans le dépôt de l'unité 1 .

b) une érosion liée à un événement plus étendu dans le temps tel qu'une baisse du niveau marin. Le surcreusement de la cuvette serait dans ce cas là essentiellement dû à sa localisation : à la conjonction de 3 facteurs pouvant influencer l'hydrodynamique locale. Les figures 5 et 6 montrent que cette cuvette d'érosion est située juste à l'extrémité de l'éperon rocheux du môle du port militaire, au débouché du cours de la rivière Penfeld et en vis-à-vis du banc sableux de St Pierre. Comme il n'y a pas d'autres profils montrant un tel faciès, on pourrait imaginer que les conditions hydrodynamiques favorisent, à cet endroit précis, l'érosion de la couverture sédimentaire sous l'action d'un ressaut hydraulique par exemple.

Cette seconde hypothèse, qui ne fait pas appel à un événement catastrophique mais seulement à des conditions hydrodynamiques spéciales en un point, probablement réalisées lorsque le niveau marin est redescendu sous un certain seuil nous semble la plus vraisemblable. Elle semble confortée par deux points : la localisation unique de cette cuvette d'érosion, et la profondeur de celle-ci qui atteint les 4,5 m et s'arrête sur le schiste. Il nous semble en effet peu probable d'atteindre $5 \mathrm{~m}$ d'érosion en une seule tempête dans un milieu aussi protégé. Et si tel est le cas, de n’observer alors qu'une seule dépression sur toute la rade.

Phase 3 : le dépôt de l'unité U2 ne s’observe qu'au sein de cette dépression : il est donc difficile de conclure quant au mode de dépôt de cette unité. Les figures 3, 4 et 5 montrent que cette unité faite d'une alternance de lits sableux et graviers (MENARD, 1967) présente des ondulations en raison de la forte exagération verticale (x 25) qui nappent la dépression. Ces ondulations provoquent des décalages de 2 à $4 \mathrm{~m}$, qui sont exactement situés dans la même zone que les failles relevées par MENARD (1967). 
Apport de la sismique marine très haute résolution à l'étude de la rade abri de Brest (France) : 1.9
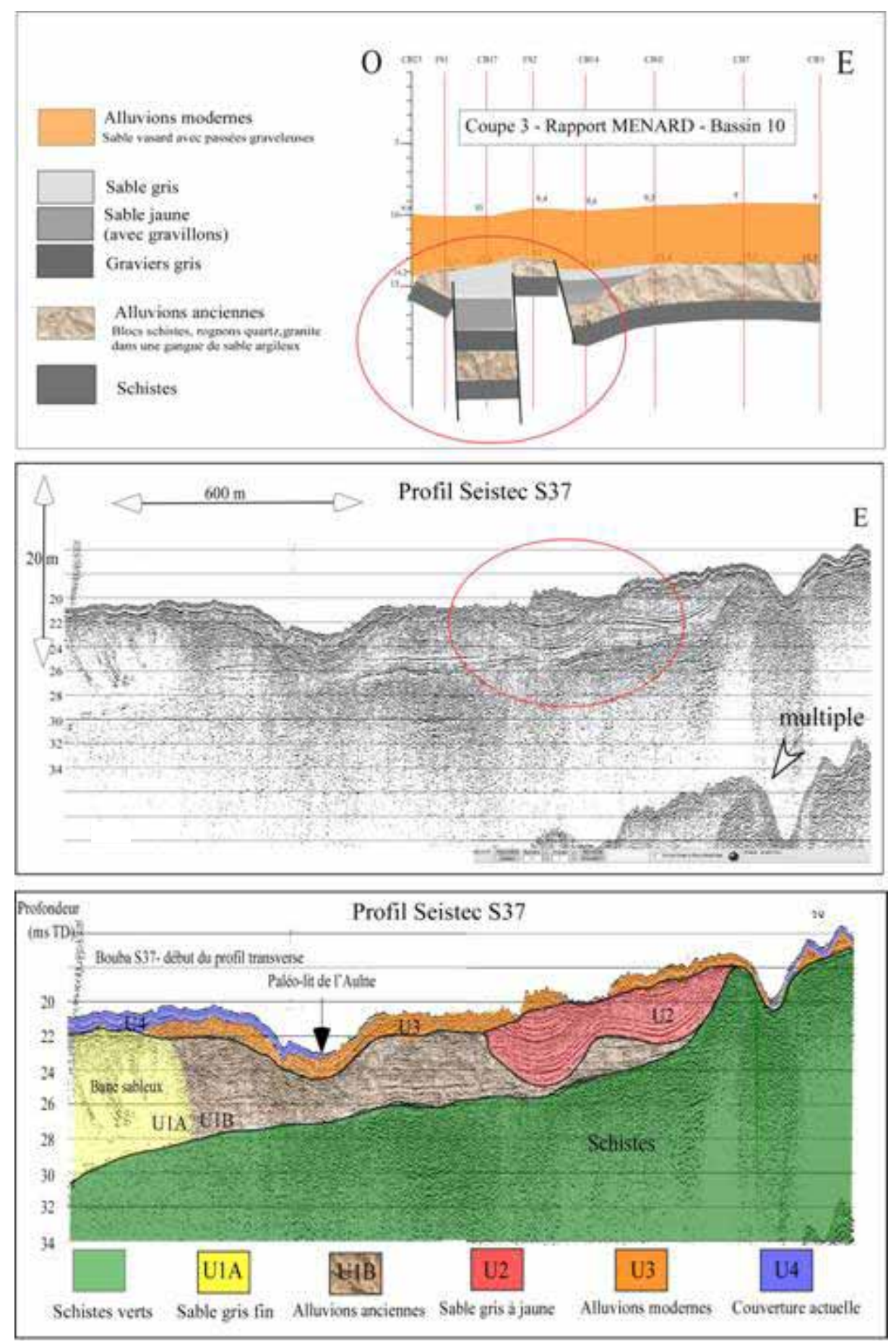

Figure 3. Comparaison du profil Seistec S37 avec la coupe géologique 3 (MENARD, 1967). 

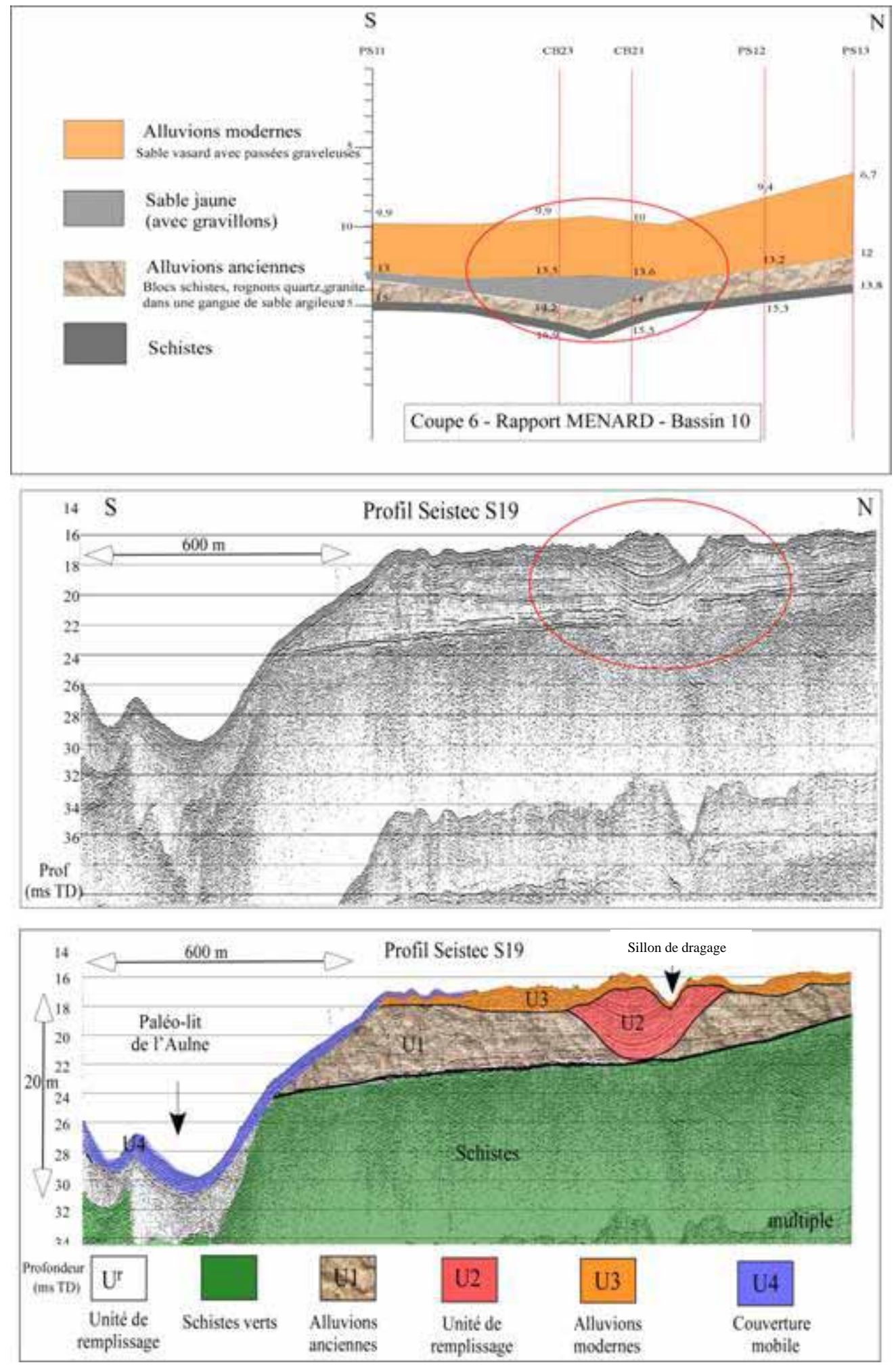

Figure 4. Comparaison du profil Seistec S19 avec la coupe géologique 6 (MENARD, 1967). 
Apport de la sismique marine très haute résolution à l'étude de la rade abri de Brest (France) : 1.11

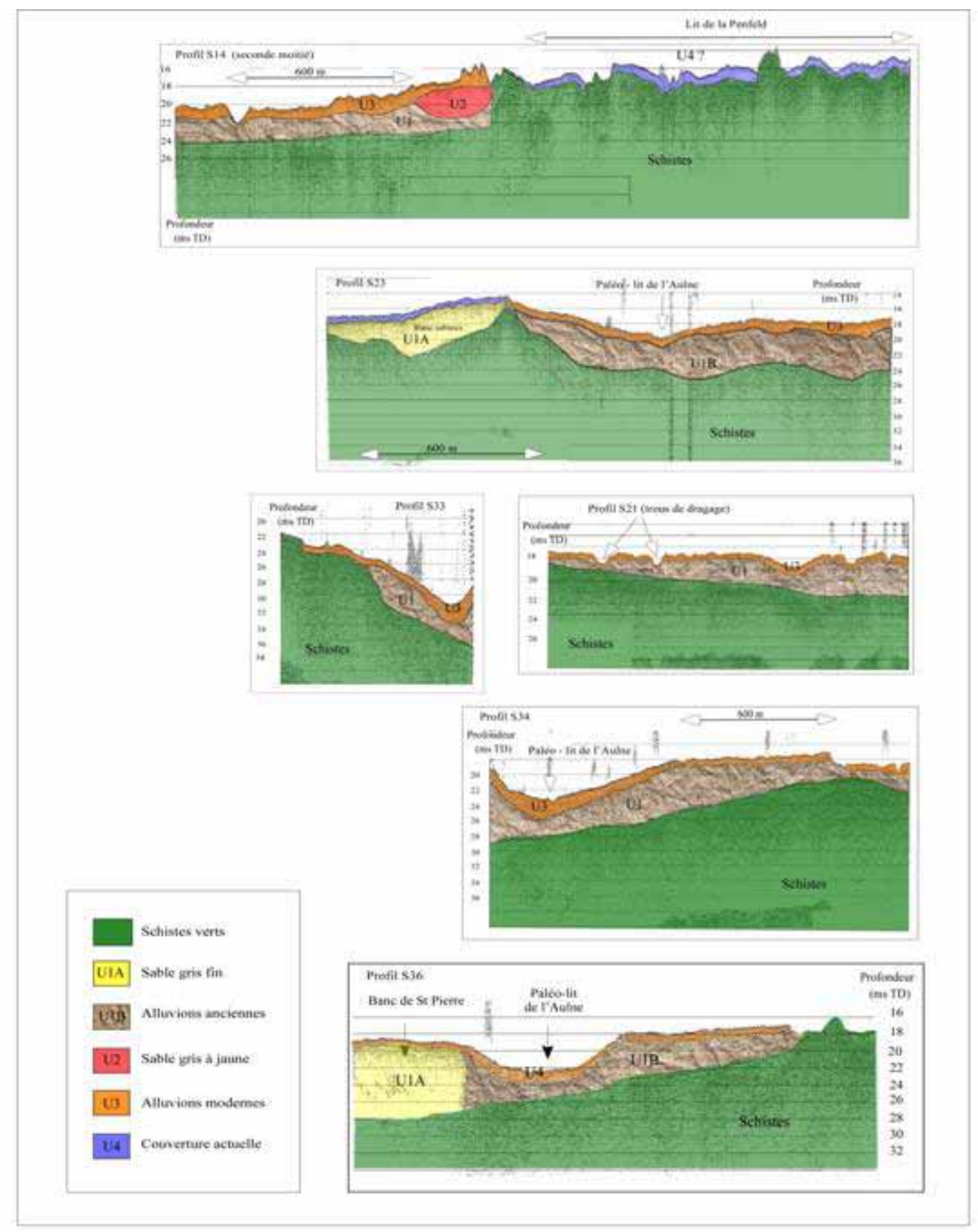

Figure 5. Interprétation de différents profils dans le port (localisation des profils indiquée sur la figure 1). 
1.12 : Revue Paralia - Vol. 3 (2010)

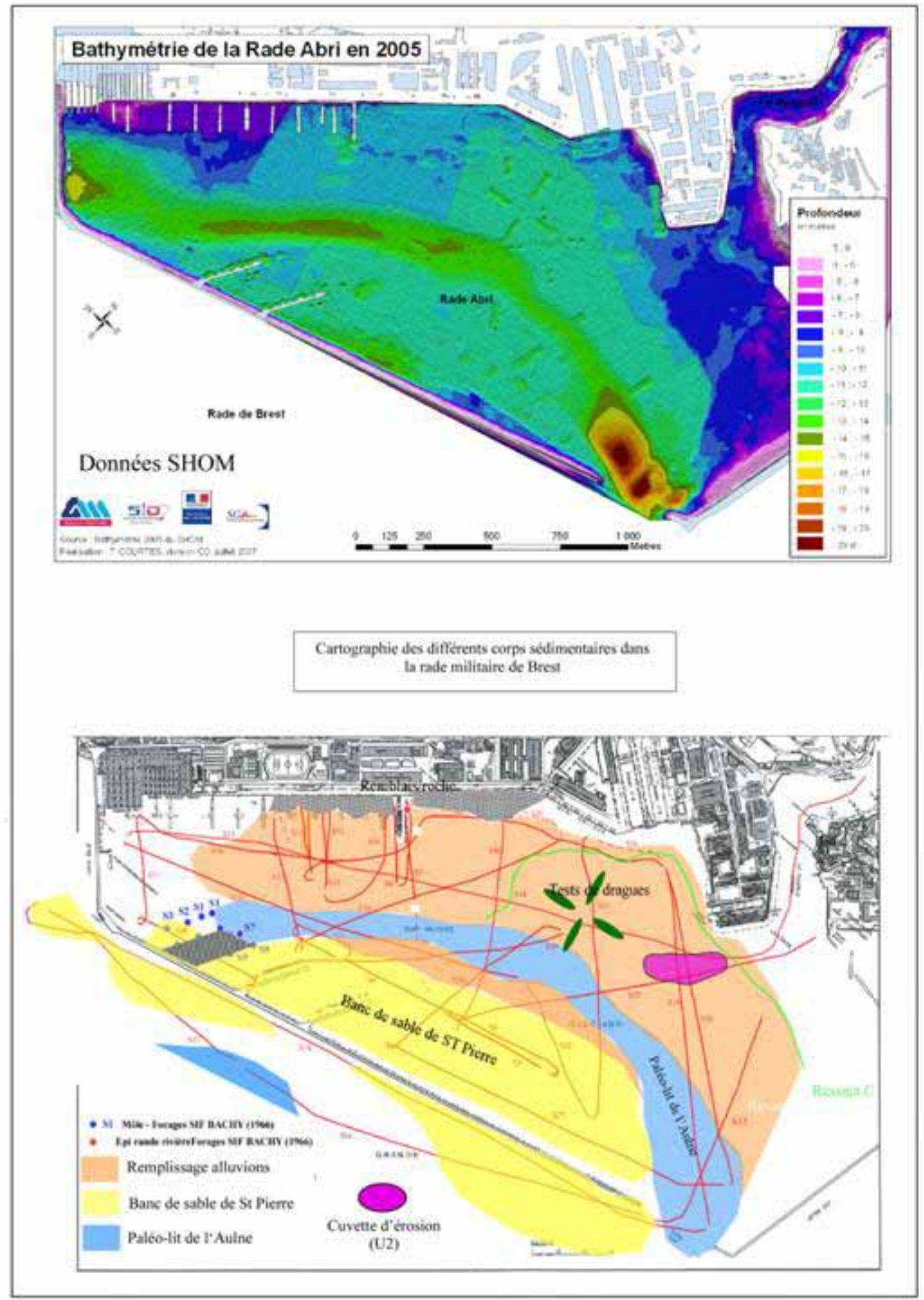

Figure 6. Interprétation des structures sédimentaires. 
Phase 4 : La quatrième phase de remplissage proprement dite, reconnue à l'échelle de la rade est composée par les alluvions modernes évoquées par Ménard (U3) atteignant quelques mètres d'épaisseur. Elle correspond à la mise en place du système hydrodynamique actuel à la faveur de la dernière phase de remontée du niveau marin, probablement vers 6000 ans BP, dominée par l'influence tidale.

Enfin, un dépôt superficiel de sables et vases en couverture mobile (U4) d'une épaisseur de 30 à $150 \mathrm{~cm}$ nappe les précédentes unités. Cette couverture qui correspond à la sédimentation actuelle est entaillée par plusieurs essais de dragages (figure 6).

Il faut souligner, qu'étant donnée l'action anthropique marquée dans cette rade militaire (dragages de zones entières pour maintenir une profondeur fixée), il est délicat d'essayer de quantifier les remplissages et d'en tirer des taux de sédimentation.

\section{Scénario 2 :}

L'unité U1 serait antérieure à l'Unité Ur, et constituerait une terrasse fluviale construite par l'Aulne antérieure à la chute du niveau marin (40000 ans BP ?). Lors de la remontée du niveau marin (à partir de 18000 ans BP) l'Unité $\mathrm{U}^{\mathrm{r}}$ pourrait se déposer. Elle serait alors soit du même âge que l'unité U2, soit située chronologiquement entre U1 et U2. Il est cependant très difficile d'attribuer le faciès U2 à un paléo lit de la Penfeld, car celle-ci est connue pour son très faible apport sédimentaire (MUSSET, 1934).

\section{Conclusions}

La réalisation de cette reconnaissance géophysique à l'aide du boomer Seistec nous a permis de proposer une reconstitution du fonctionnement sédimentaire de la rade abri du port militaire de Brest. Par exemple, l'influence de l'Aulne, et non de la Penfeld comme le laissait supposer la disposition actuelle, s'est avérée prépondérante dans la dynamique sédimentaire passée de la rade abri. Le comblement sédimentaire s'est déroulé en 4 phases de dépôt, il a été interrompu par une phase d'érosion marquée probablement lors d'un épisode de baisse du niveau marin.

Enfin, cette étude géophysique nous a permis d'appréhender de façon différente des structures géologiques attribuées à des failles, et de les associer à la présence d'une cuvette d'érosion remarquable. Cette dépression atteignant le toit du schiste (surcreusement sur une épaisseur de presque $5 \mathrm{~m}$ ) est localisée en une seule zone, à la conjonction de facteurs hydrauliques favorables puis a été comblée lors d'une nouvelle phase de remplissage de la baie. La combinaison de deux approches associant la vision continue à la vision ponctuelle a rendu possible la compréhension de cette structure particulière. 


\section{Références bibliographiques}

BALTZER A., STEPANIAN A., OWONO J., TESSIER B. (2003). Identification des sables vacuolaires à terre et en mer. Revue Française de Génie Civil, n 9, Vol. 7, pp 1139-1149.

FUGRO (1999). Rapport N FR 98 Gy Co 1002 Revue B pour le Port Autonome du Havre

HALLEGOUET B. (1989). La Presqu'Ile de Crozon : évolution géomorphologique. Historien-Géographe, 318, pp 141-148.

JOUET G., AUGRIS C., HALLEGOUET B., LE ROY P., ROLET J. (2003). La Vallée d'Ys ; un paléoréseau hydrographique immergé en Baie de Douarnenez (Finistère, France). Comptes Rendus Geoscience, pp 487-494.

MARTY N. (1994). Apports de la sismique très haute résolution à l'étude du système de paléovallées en rade de Brest. DEA, Université de Bretagne Occidentale, Brest, 41 p.

MENIER D., REYNAUD J-Y., PROUST J-N., GUILLOCHEAU F., GUENNOC P., TESSIER B., BONNET S., GOUBERT E. (2006). Inherited fault control on the drainage pattern and infilling sequences of late glacial incised valleys, SE coast of Brittany, France. SEPM special publication, $n^{\circ}$ 85, pp 37-55.

MENARD L. (1967). Rapport d'étude des sols et fondations pour le Port Militaire de Brest. 21 p.

MITCHUM J.R., VAIL P.R., SANGREE J.B. (1977). Stratigraphy interpretation of seismic reflection pattern in depositional sequences. In Payton C.E. Eds Seismic Stratigraphy. Applications to Hydrocarbon exploration. American Association of Petroleum Geology, 26, pp 117-134.

MOSHER D.C., SIMPKIN P. (1999). Status and trends of marine high resolution seismic reflection profiling data acquisition. Geosciences Canada, $22 \mathrm{p}$.

MUSSET P. (1934). La formation du réseau hydrographique de la Bretagne Occidentale. Ann. Geogr 246, pp 561-578.

PROUST J-N., MENIER D., GUILLOCHEAU F., GUENNOC P., BONNET S., ROUBY D., LE CORRE C. (2001). Les vallées fossiles de la baie de la Vilaine : nature et évolution du prisme sédimentaire côtier du Pléistocène armoricain. Bulletin de la Société géologique de France 172 6, pp 737-749.

SIF BACHY (1966). Rapport pour la Marine Nationale pour le Port de Brest.

SIMPKIN P., DAVIS M. (1993). Study for seismic profiling in very shallow water, a novel receiver, Vol. 34, 5 p.

SIMPKIN P. (2005). The boomer soundsource as a tool for shallow water geophysical exploration. Marine Geophysical Researches 26, pp 171-181. 